\title{
ON THE SCHUR MULTIPLIER OF A QUOTIENT OF A DIRECT PRODUCT OF GROUPS
}

\author{
Graham Ellis
}

We use a nonabelian exterior product to strengthen two old and basic results on the

Schur multiplier of a (central) quotient of a direct product of groups.

This is one of a series of papers (see also $[5,6,7,8]$ ) advertising the relevance of a certain 'nonabelian exterior product.' to the development and exposition of the basic theory of the Schur multiplier of a group. We shall use the exterior product to prove the following generalisation of a result of Eckmann, Hilton and Stammbach [3].

TheOREM 1. Let $A=M \times N$ be a direct product of groups, let $\pi_{M}: A \rightarrow M$, $\pi_{N}: A \rightarrow N$ be the projections, and let $U$ be a normal subgroup of $A$. Set $G=A / U$, $\bar{M}=M / \pi_{M} U, \quad \bar{N}=N / \pi_{N} U$. The Schur multiplier $H_{2}(G)$ fits into a short exact sequence

$$
0 \rightarrow B \rightarrow H_{2}(G) \rightarrow \frac{U \cap[A, A]}{[U, A]} \rightarrow 0
$$

where $B$ is an Abelian group that fits into exact sequences

$$
\begin{gathered}
{[U, A]_{a b} \oplus H_{2}(M) \oplus H_{2}(N) \rightarrow B \rightarrow \bar{M}_{a b} \otimes \bar{N}_{a b} \rightarrow 0} \\
M_{a b} \otimes N_{a b} \rightarrow B \rightarrow \operatorname{ker}\left(H_{2}(\bar{M}) \rightarrow \frac{\pi_{M} U}{\left[M, \pi_{M} U\right]}\right) \oplus \operatorname{ker}\left(H_{2}(\bar{N}) \rightarrow \frac{\pi_{N} U}{\left[N, \pi_{N} U\right]}\right) .
\end{gathered}
$$

A special case of this theorem, in which $U$ is assumed to be central in $A$, was proved in [3]. As illustrated in [3], the theorem can be viewed as a tool for determining some of the structure of the Schur multiplier $H_{2}(G)$ from a knowledge of $H_{2}(A)$.

Theorem 1 also implies a result of Wiegold [9] which states that if $U \cong \pi_{M} U \cong \pi_{N} U$, if $U$ is central in $A$, and if $G$ is finite, then $\bar{M}_{a b} \otimes \bar{N}_{a b}$ is isomorphic to a subgroup of $H_{2}(G)$. To deduce this result it in fact suffices to assume that $G$ is finite, for then $H_{2}(G)$ is finite, and thus (2) provides a surjection $B \rightarrow \bar{M}_{a b} \otimes \bar{N}_{a b}$ of finite groups. So $\bar{M}_{a b} \otimes \bar{N}_{a b}$ must be isomorphic to a subgroup of $B$, and hence isomorphic to a subgroup of $H_{2}(G)$.

Received 13th May, 1998

Copyright Clearance Centre, Inc. Serial-fee code: 0004-9729/98 \$A2.00+0.00. 
We shall show how Wiegold's result can be reworked into the following slightly more general proposition.

Proposition 2. Let $M, N$ be normal subgroups of a group $K$ such that $[M, N]=1$. Set $G=M N$ and suppose that the image of the canonical homomorphism $\phi: G \rightarrow K_{a b}$ is a direct summand of $K_{a b}$, that is $K_{a b} \cong \phi(G) \oplus(K / G)_{a b}$. Then:

(i) $(\phi(M) / \phi(M \cap N)) \otimes(\phi(N) / \phi(M \cap N))$ is isomorphic to a quotient of $H_{2}(K)$;

(ii) if $M_{a b}$ and $N_{a b}$ are finite then $(\phi(M) / \phi(M \cap N)) \otimes(\phi(N) / \phi(M \cap N))$ is isomorphic to a subgroup of $\mathrm{H}_{2}(K)$.

Note that if $K=M N$ then $(\phi(M) / \phi(M \cap N)) \cong(M / M \cap N)_{a b}$ and $(\phi(N) /$ $\phi(M \cap N)) \cong(N / M \cap N)_{a b}$.

For the proof of Theorem 1 we recall from $[2,4]$ that any group $E=P Q$, which is a product of two normal subgroups $P, Q \unlhd E$, gives rise to a natural exact sequence

(4) $\operatorname{ker}(P \wedge Q \rightarrow[P, Q]) \rightarrow H_{2}(E) \rightarrow H_{2}(E / P) \oplus H_{2}(E / Q) \rightarrow \frac{P \cap Q \cap[E, E]}{[P, Q]} \rightarrow 0$.

The derivation given in [4] is purely algebraic and uses only elementary arguments based on Hopf's formula for the Schur multiplier and on an isomorphism

$$
H_{2}(E) \cong \operatorname{ker}(E \wedge E \stackrel{\lambda}{\rightarrow} E) \text {. }
$$

The exterior product $P \wedge Q$ is the group generated by symbols $x \wedge y(x \in P, y \in Q)$ subject to the relations

$$
\begin{gathered}
x x^{\prime} \wedge y=\left(x x^{\prime} x^{-1} \wedge x y x^{-1}\right)(x \wedge y), \\
x \wedge y y^{\prime}=(x \wedge y)\left(y x y^{-1} \wedge y y^{\prime} y^{-1}\right), \\
z \wedge z=1
\end{gathered}
$$

for $x, x^{\prime} \in P, y, y^{\prime} \in Q, z \in P \cap Q$. The homomorphism $\lambda$ is defined on generators by $\lambda(x \wedge y)=x y x^{-1} y^{-1}$.

On taking $E=A, P=U$ and $Q=A$, sequence (4) reduces to an exact sequence

$$
\operatorname{ker}(U \wedge A \stackrel{\wedge}{\rightarrow}[U, A]) \stackrel{\beta}{\rightarrow} H_{2}(A) \stackrel{a}{\rightarrow} H_{2}(G) \rightarrow \frac{U \cap[A, A]}{[U, A]} \rightarrow 0 .
$$

We set $B=\operatorname{coker}(\beta)=\operatorname{im}(\alpha)$ and note that this definition of $B$ leads to the exact sequence (1).

If $P, Q \leqslant E$ are such that $[P, Q]=1$ then it is readily shown (see [2] for details) that

$$
\operatorname{ker}(P \wedge Q \stackrel{\mathcal{A}}{\rightarrow}[P, Q]) \cong P_{a b} \otimes Q_{a b} / \Delta
$$


where $\Delta$ is the subgroup of $P_{a b} \otimes Q_{a b}$ generated by the tensors $z[P, P] \otimes z[Q, Q]$ for $z \in P \cap Q$. We set

$$
P_{a b} \wedge Q_{a b}=P_{a b} \otimes Q_{a b} / \Delta .
$$

The naturality of sequence (4) and the isomorphisms $G / M \cong \bar{N}, G / N \cong \bar{M}$ lead to the following commutative diagram in which the rows and columns are exact.

$$
\begin{aligned}
& 0 \rightarrow \quad M_{a b} \otimes N_{a b} \quad \rightarrow H_{2}(A) \rightarrow \quad H_{2}(M) \oplus H_{2}(N) \quad \rightarrow \quad 0 \\
& \begin{array}{cccc}
\downarrow & \downarrow & \downarrow \alpha & \\
(M U / U)_{a b} \wedge(N U / U)_{a b} & \rightarrow \quad H_{2}(G) & \rightarrow & H_{2}(\bar{M}) \oplus H_{2}(\bar{N})
\end{array} \\
& \downarrow \\
& 0 \\
& \frac{\pi_{M}(U)}{\left[M, \pi_{M} U\right]} \oplus \frac{\pi_{N}(U)}{\left[N, \pi_{N} U\right]}
\end{aligned}
$$

The exact sequence (3) follows immediately from this diagram.

In order to derive sequence (2) note that the composition of the inclusion $H_{2}(M) \oplus$ $H_{2}(N) \stackrel{\iota}{\hookrightarrow} H_{2}(A)$ with the surjection $H_{2}(A) \stackrel{\leftrightarrow}{\rightarrow} B$ yields a map with cokernel

$$
\begin{aligned}
\operatorname{coker}\left(H_{2}(M) \oplus H_{2}(N) \stackrel{\alpha}{\rightarrow} B\right) \\
=\operatorname{coker}\left(H_{2}(M) \oplus H_{2}(N) \oplus \operatorname{ker}(U \wedge A \stackrel{\wedge}{\rightarrow}[U, A]) \stackrel{\rho}{\rightarrow} H_{2}(A)\right) .
\end{aligned}
$$

The natural isomorphism (5) leads to a commutative diagram

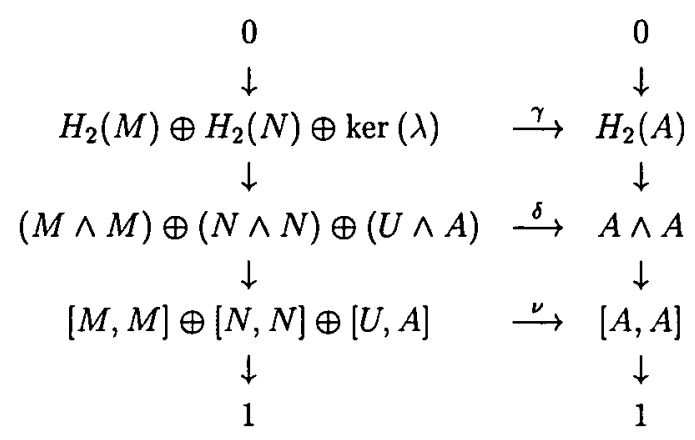

in which the columns are exact. Note that $\operatorname{coker}(\nu)=0$ and $\operatorname{coker}(\delta)=\bar{M}_{a b} \otimes \bar{N}_{a b}$. (To see the latter equality, recall [1] that $A \wedge A \cong(M \wedge M) \oplus(N \wedge N) \oplus\left(M_{a b} \otimes N_{a b}\right)$, and note that if $(x, y) \in U \leqslant M \times N$ and $(a, b) \in M \times N$ then working in $A \wedge A$ we have

$$
x y \wedge a b=\left(y \wedge{ }^{x} a\right)(y \wedge b)(x \wedge a)\left({ }^{a} x \wedge b\right) .
$$

Thus

$$
\operatorname{coker}(\delta) \cong M_{a b} \otimes N_{a b} / \Gamma
$$


where $\Gamma$ is the subgroup of $M_{a b} \otimes N_{a b}$ generated by the elements $u[M, M] \otimes b[N, N]$ and $a[M, M] \otimes v[N, N]$ for $a \in M, b \in N, u \in \pi_{M} U, v \in \pi_{N} U$. It follows that $\operatorname{coker}(\delta)=\bar{M}_{a b} \otimes \bar{N}_{a b}$.) Diagram (7) yields an exact sequence

$$
\rightarrow \operatorname{ker}(\nu) \rightarrow \operatorname{coker}(\gamma) \rightarrow \operatorname{coker}(\delta) \rightarrow \operatorname{coker}(\nu)
$$

which we recognise as

$$
\rightarrow[U, A] \rightarrow \operatorname{coker}(\alpha \iota) \rightarrow \bar{M}_{a b} \otimes \bar{N}_{a b} \rightarrow 0 .
$$

The exact sequence (2) follows from this sequence and the fact that coker $(\alpha \iota)$ is Abelian.

Let us now turn to the proof of Proposition 2. The quotient homomorphism

$$
K \rightarrow(K / M \cap N)_{a b} \cong \phi(M) / \phi(M \cap N) \oplus \phi(N) / \phi(M \cap N) \oplus(K / G)_{a b}
$$

induces a homology homomorphism

$$
\begin{gathered}
H_{2}(K) \rightarrow H_{2}\left(\phi(M) / \phi(M \cap N) \oplus \phi(N) / \phi(M \cap N) \oplus(K / G)_{a b}\right) \cong \\
H_{2}(\phi(M) / \phi(M \cap N)) \oplus H_{2}\left(\phi(N) / \phi(M \cap N) \oplus(K / G)_{a b}\right) \oplus \\
(\phi(M) / \phi(M \cap N) \otimes \phi(N) / \phi(M \cap N)) \oplus\left(\phi(M) / \phi(M \cap N) \otimes(K / G)_{a b}\right) .
\end{gathered}
$$

By projecting onto the penultimate summand we obtain a homomorphism

$$
\rho: H_{2}(K) \rightarrow \phi(M) / \phi(M \cap N) \otimes \phi(N) / \phi(M \cap N) .
$$

The homomorphism $\rho$ is surjective because the condition $[M, N]=1$ implies there is a surjective composite homomorphism

$$
M_{a b} \wedge N_{a b} \cong M \wedge N \stackrel{\leftrightarrow}{\rightarrow} H_{2}(K) \stackrel{\rho}{\rightarrow} \phi(M) / \phi(M \cap N) \otimes \phi(N) / \phi(M \cap N) .
$$

(The isomorphism follows from (6), and the homomorphism $\mu$ is derived from (4).) This proves part (i) of Proposition 2. If $M_{a b}$ and $N_{a b}$ are finite then so too is $M_{a b} \wedge N_{a b}$; hence $\operatorname{im}(\mu)$ is finite and thus contains a subgroup isomorphic to its quotient $\phi(M) / \phi(M \cap N) \otimes$ $\phi(N) / \phi(M \cap N)$. This proves part (ii) of Proposition 2.

\section{REFERENCES}

[1] R. Brown, D.L. Johnson and E.F. Robertson, 'Some computations of nonabelian tensor products of groups', J. Algebra 111 (1987), 177-202.

[2] R. Brown and J.-L. Loday, 'Van Kampen theorems for diagrams of spaces', Topology 26 (1987), 311-335.

[3] B. Eckmann, P.J. Hilton and U. Stammbach, 'On the Schur multiplicator of a central quotient of a direct product of groups', J. Pure Appl. Algebra 3 (1973), 73-82. 
[4] G. Ellis, 'Nonabelian exterior products of groups and an exact sequence in the homology of groups', Glasgow Math. J. 29 (1987), 13-19.

[5] G. Ellis, 'Capability, homology, and central series of a pair of groups', J. Algebra 179 (1995), 31-46.

[6] G. Ellis, 'The Schur multiplier of a pair of groups', Appl. Categ. Structures (to appear).

[7] G. Ellis, 'On the capability of groups', Proc. Edinburgh Math. Soc. (to appear).

[8] G. Ellis, 'A bound for the derived and Frattini subgroups of a prime-power group', Proc. Amer. Math. Soc. 126 (1998), 2513-2523.

[9] J. Wiegold, 'Some groups with nontrivial multiplicators', Math. Z. 120 (1971), 307-308.

Department of Mathematics

National University of Ireland

Galway

Ireland

e-mail: graham.ellis@ucg.ie
Current address:

Max-Planck-Institut für Mathematik

Gottfried-Claren-Straße 26

53225 Bonn

Germany 